\title{
PROTON ANTIPROTON ANNIHILATION INTO MUON PAIR
}

\author{
M. Conversi, T. Massam, Th. Muller, M. Schneegans and A. Zichichi \\ CERN, Geneva, Switzerland
}

(Presented by A. ZICHICHI)

The experiment I wish to talk about refers to the study of the process:

$$
\bar{p}+p \rightarrow \bar{\mu}+\mu \text {. }
$$

As is well known the interest of this experiment is twofold:

i) determination of the electromagnetic structure of the proton in the time-like region;

ii) check of the equality of the electromagnetic properties of the muon and the electron for time-like momentum transfers.
The experiment has been performed at CERN, using the new high intensity separated $\bar{p}$-beam [1] whose characteristics are probably worth recording:

with $50 \%$ of the total circulating proton beam of the CERN Proton-Syncrotron (which has been during the last week $10^{12}$ protons/pulse) we get $50000 \frac{\bar{p}}{\text { burst }}$ with a $\pi$-contamination: $\frac{\pi}{\bar{p}}=3.5 ; 250 \mathrm{~m} \mathrm{sec}$. spilling time and

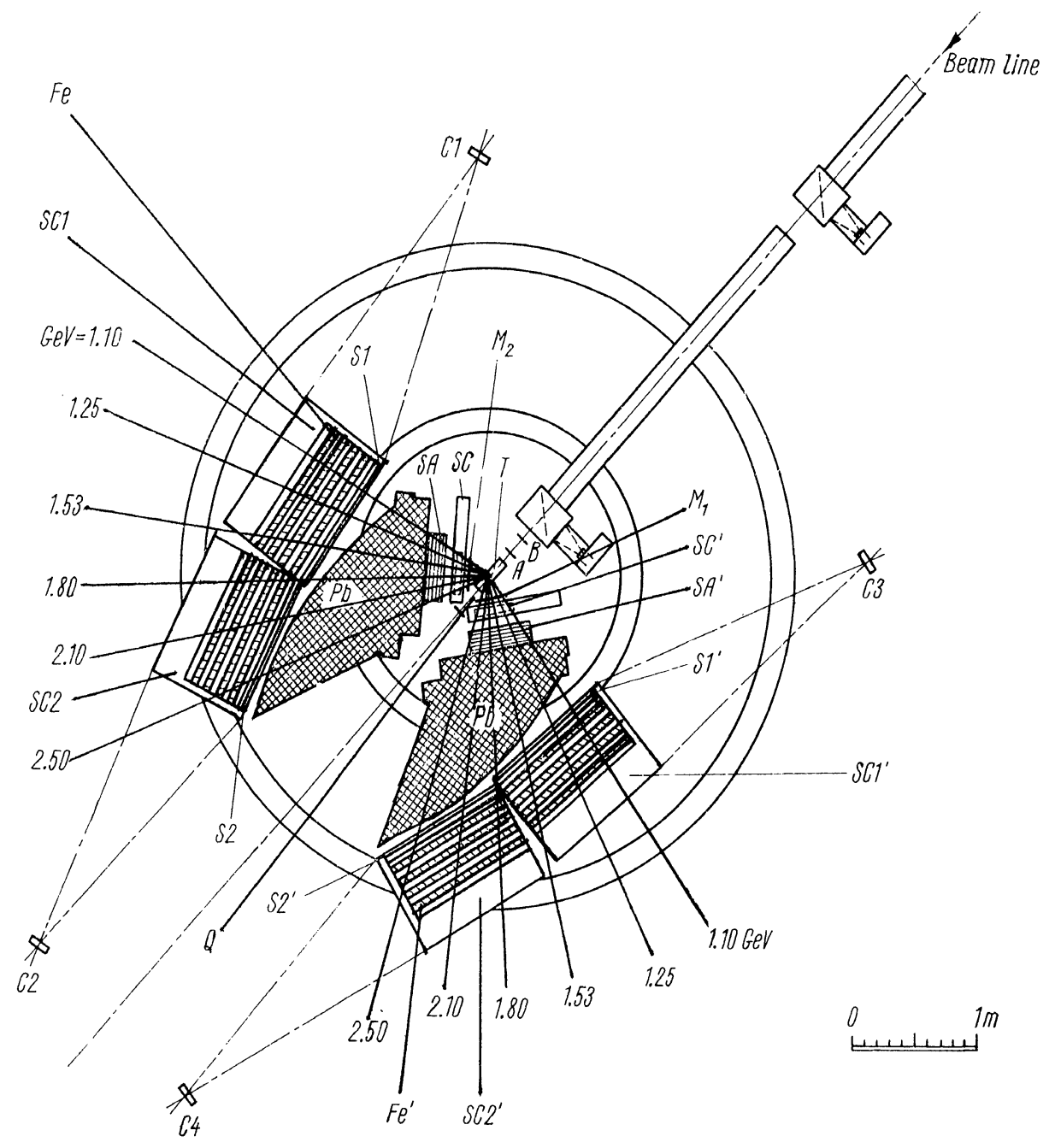

Fig. 1. Experimental set-up. 
$2.3 \mathrm{sec}$ repetition rate. We want to emphasize that it has been impossible for scheduling reasons to make an absolute calibration of our $\bar{p}$-intensity per circulating proton but we do not expect our figure to be oft by more than $30 \%$.

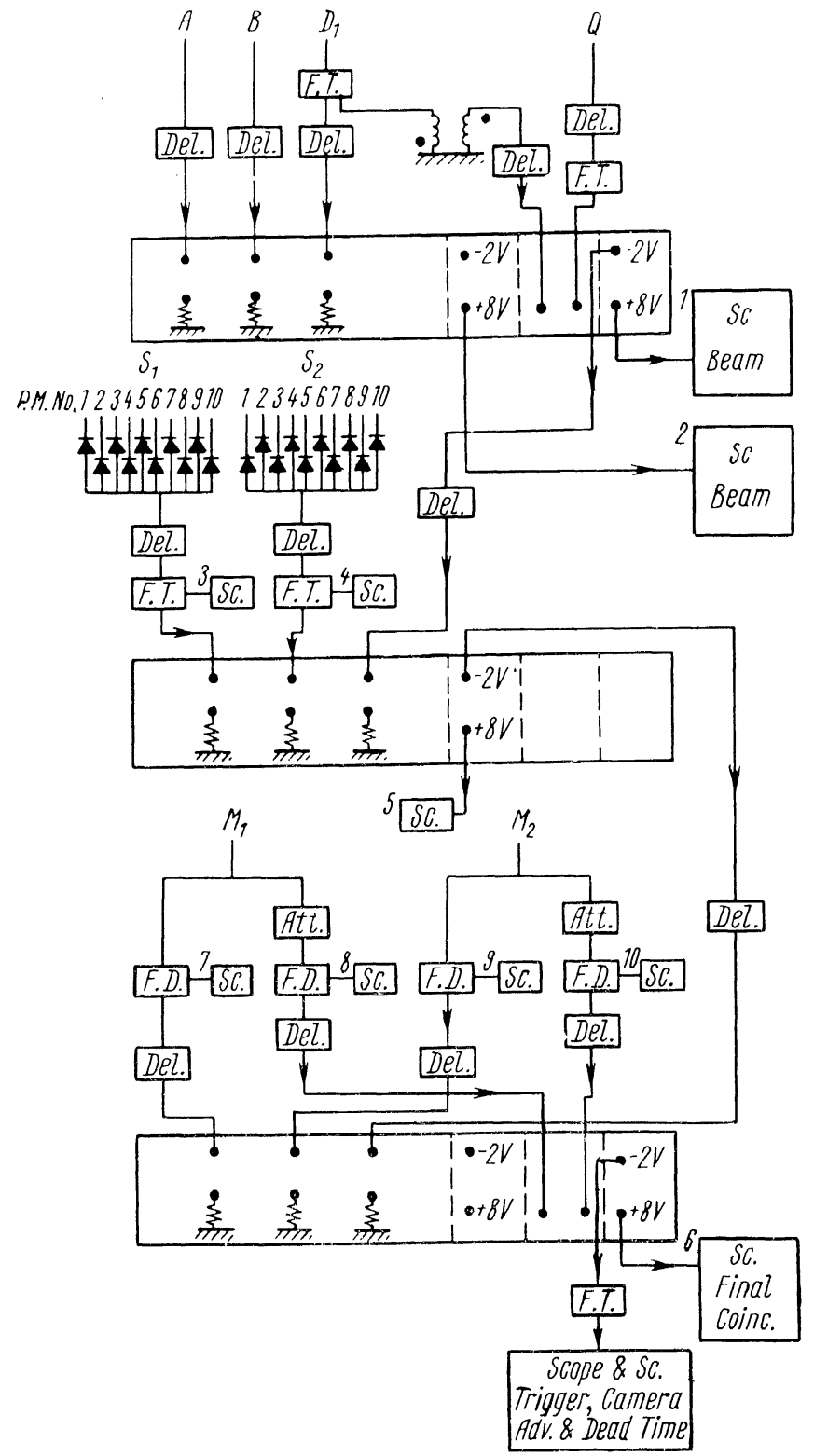

Fig. 2. Electronic block diagram.

All we can say with confidence is that we were able to collect $1.88 \times 10^{9} \quad \bar{p} /$ day under the conditions of machine operation specified above.

The $\bar{p}$-beam momentum was chosen to be $2.5 \mathrm{GeV} / \mathrm{c}$; this value being the one that maximize the expected rate for process 1 ). The corresponding invariant time-like four-momentum transfer is $q^{2}=6.8(\mathrm{Gev} / \mathrm{c})^{2}$.
Let me recall you the two relevant figures concerning cross sections. If the proton is point like the expected cross section for process 1) at $2.5 \mathrm{Gev} / \mathrm{c}$ incident momentum is $\sigma_{\bar{p}+p \rightarrow \bar{\mu}+\mu}=$ $=242$ nanobarns $*$. This cross section should be compared with the correspondent total annihilation cross section, which is

$$
\sigma_{\bar{p}+p} \rightarrow \text { total annihilation } \simeq 5 \times 10^{7}
$$

nbarns in order to realize the rejection needed to discriminate against $\pi$ 's.

Fig. 1 shows the experimental apparatus which consists of:

i) a beam telescope: a threshold gas Čerenkov counter $D$, and two beam-defining plastic scintillator counters $A$ and $B$;

ii) two identical telescopes whose function is to select muons and to reject pions. The details of this telescope are described elsewhere [2].

Fig. 2 shows a simplified electronic block diagram, which essentially consists of a beamtelescope in fast coincidence with the muonsignatures from both left and right telescopes. A coincidence between [(Beam) $\times$ (telescope left $\times$ (telescope right)] will trigger our spark chambers in which we identify the muons by range measurements [3].

The experiment has finished few days before this Conference started. The results are therefore preliminary and can be stated as follows: If the cross section is point like we should have in our sample 200 muon-pair-events we do not have in our sample more than 30 candidates by experience we know that the rejection on this number is not going to be less than a factor of four. Therefore our present limit on the cross section for process 1) is $\sigma_{\bar{p} p \rightarrow \bar{\mu} \mu} \leqslant 10 \mathrm{nb}$.

When compared with the point like cross section this gives

$$
\frac{\sigma_{\bar{p} p}^{\exp } \rightarrow \bar{\mu} \mu}{\sigma_{\bar{p} p \rightarrow \bar{\mu} \mu}^{\text {point like }}} \leqslant 5 \times 10^{-2} .
$$

It follows that we confirm with the muon channel (process 1$)$ the results already obtained with the electron channel $(\bar{p} p \rightarrow \bar{e})$ [4] namely the existence of a big time-like electromagnetic structure of the proton for a value of the invariant 4-momentum transfer equal to 6.8 $(\mathrm{GeV} / \mathrm{c})^{2}$.

* Nanobarn-10-33 $\mathrm{cm}^{2}$. 


\section{REFERENCES}

1. B r a u t $t \mathrm{i}$ G. et al. A high intensity separated beam of $\pi, \mathrm{K}$ and $p s$. Presented by A. Zichichi at this Conference.

2. $(\pi-\mu)$ discrimination at high energy Massam $\mathrm{T}$. et al. CERN-yellow Report - 1964 .

3. Range Measurements for muons in the Gev Region Buhler A. et al. Nuovo cimento (1964), to be published.

4. C o n vers i $M$. et al. Proceedings of the International Conference on Elementary Particles, SIENA, October 1963.

\section{DISCUSSION}

$\mathrm{Nguyen} \mathrm{Van} \mathrm{Hieu}$

My remark refers to a paper by Tinlot and Zichichi. Recently, in a work of Prof. A.A. Logunov and other
Dubna theoreticians it was shown that the form factor $\left(F_{i}(t)\right.$ of nucleons in annihilation processes $t>0$ and in scattering processes $t<0$ must have asymptotes which coincide with

$$
\lim _{t \rightarrow \infty}\left|\frac{F_{i}(t)}{F(-t)}\right|=1
$$

This asymptotic equality is a consequence of the general analytical properties of form factors. Consequently, it is quite possible to measure the form factors and to experimentally verify this equality.

For the annihilation processes the form factors are complex. With the help of the method A.A. Logunov et al. it can be shown that the phase of two form factors (electric and magnetic) are equal to one another at the asymptote and this mutual phase is determined by the behavior of the modulus of the form factors. 